\title{
The infernal business of contract cheating: understanding the business processes and models of academic custom writing sites
}

\author{
Cath Ellis ${ }^{1 *}$ (D) lan Michael Zucker ${ }^{1}$ and David Randall ${ }^{2}$
}

\author{
* Correspondence: \\ cath.ellis@unsw.edu.au \\ ${ }^{1}$ Faculty of Arts and Social Sciences, \\ UNSW, Sydney, NSW 2052, Australia \\ Full list of author information is \\ available at the end of the article
}

\begin{abstract}
While there is growing awareness of the existence and activities of Academic Custom Writing websites, which form a small part of the contract cheating industry, how they work remains poorly understood. Very little research has been done on these sites, probably because it has been assumed that it is impossible to see behind their firewalls and password protection. We have found that, with some close scrutiny, it is indeed possible to find some 'cracks' in these sites through which we can look to gain insights into the business processes that operate within them. We have reverse engineered the business processes that operate within some of these sites. From this we have also been able to identify three different business models that are supported by these sites. Our analysis supports important findings about how these sites operate that can be used to inform future strategies to detect and deter contract cheating.

Keywords: Contract cheating, Business model and academic integrity
\end{abstract}

\section{Background}

Contract cheating occurs when a student procures a third party (who knows about and benefits from the transaction) to produce academic work (that is usually, but not always assessable work) that the student then submits to an educational institution as if it were their own. It constitutes a form of plagiarism: presenting someone else's words and/or ideas as your own without appropriate attribution. The contract can be either paid or unpaid; paid work obviously involves payment in money, but unpaid work could involve favours and other forms of mutual obligation (Lancaster and Clarke 2014a). While there is a widespread perception that contract cheating is a recent phenomenon, as Bertram Gallant points out in her history of academic misconduct, it is probably one of the oldest forms of cheating there is (Bertram Gallant 2008). The way that contract-cheating transactions occur, however, has changed over time. The most recent changes have been a result of emerging technologies that facilitate new ways to procure and produce bespoke academic work.

It is likely that contract cheating is not well understood by many of the academic staff responsible for marking and administering student academic work in educational institutions. This is hardly surprising: contract cheating is, by its very nature, covert and duplicitous. Along side this low level of awareness, there are not, as yet, tools that

(c) The Author(s). 2018 Open Access This article is distributed under the terms of the Creative Commons Attribution 4.0 International License (http://creativecommons.org/licenses/by/4.0/), which permits unrestricted use, distribution, and reproduction in any medium, provided you give appropriate credit to the original author(s) and the source, provide a link to the Creative Commons license, and indicate if changes were made. 
can effectively and efficiently detect contract cheating at the point of submission. Most institutions will have had some experience of investigating and penalising students for contract cheating and in some instances these have been reported in the media (Jacks 2016). There is a growing concern across the Higher Education sector that the number of instances being detected is considerably smaller than the volume of work being procured, produced and submitted to institutions for assessment. Concomitantly, there is almost certainly a widespread perception amongst students who are currently or who are contemplating cheating in this way that the likelihood of their being caught and penalised is very small indeed. Rigby et al. make the important point that in the contract cheating market place 'the demand for essays involves the interplay of risk, penalties and the payoffs and the ethics, norms and risk preferences of the individual facing the option to buy' (Rigby et al. 2015 p23). A low risk of detection is, therefore, almost certainly an important influencing factor for at least some students. Macdonald and Carroll are at pains to point out that detection alone is not an adequate solution to the problem of academic misconduct (Macdonald and Carroll 2006). Detection, however, can contribute in useful ways to a holistic approach to deterrence and is therefore a critical part of the management of academic integrity in any educational institution. The premise of this paper is that in order to improve detection, it is first important to understand better how the business of contract cheating works.

There are several ways in which contract cheating transactions take place. In his 2009 paper "Contract Cheating: A New Phenomenon in Cyber-Plagiarism" Mahmood divides the contract cheating problem into five segments: bespoke essay sites, using friends and family, discussion sites, tutorial sites and auction sites (Mahmood 2009). In their more recent paper, Newton and Lang also divide the problem into five sections that are slightly different to Mahmood's: academic custom writing, online labour markets, prewritten essay banks, file-sharing sites and paid exam takers (Newton and Lang 2015). This paper suggests that neither of these views provide a fully comprehensive description of the different segments of contract cheating and that it is useful to combine them to identify instead six segments: family and friends; academic custom writing sites; legitimate learning sites (eg. file sharing, discussion and microtutoring sites); legitimate non-learning sites (eg. freelancing sites and online auction sites); paid exam takers; and pre-written essay banks. It is on the second of these segments - academic custom writing sites - that this paper focuses its attention.

There is a growing body of scholarly work on contract cheating. To date the bulk of it has been undertaken by Clarke and Lancaster who have focussed their attention on the use of legitimate freelancing and auction sites for the purposes of contract cheating (see Clarke \& Lancaster, 2006, 2007, 2013; Lancaster \& Clarke, 2014a, 2014b). Wallace and Newtown have also investigated the speed of production of contract cheating procured and produced through these types of site (Wallace and Newton 2014). The scholarly work on academic custom writing sites has considered such things as the legality of the transactions (Newton and Lang 2015; Draper et al. 2017), the quality of work being produced (Lines 2016), the writers who produce the work (Bartlett 2009; Sivasubramaniam et al. 2016), the ability of markers to detect essays purchased from these sites (Dawson and Sutherland-Smith 2017) and the variety of features used by the sites to persuade students to use their services (Rowland et al. 2017). This paper offers some initial findings that have resulted from a close examination of a selection of sites associated with academic custom writing. 
This paper does not include any information that specifically identifies any of these sites in order to avoid promoting any individual companies (see Draper et al. 2017). Information relating to these sites has been made available to the reviewers and also to the Australian Tertiary Education Quality Standards Authority (TEQSA) and the Quality Assurance Agency (QAA) in the United Kingdom. The analysis is based on the examination of these sites in particular and this paper makes no claims that these findings represent the academic custom writing industry or the contract-cheating problem as a whole. The analysis does, however, offer a useful window into part of one segment of the industry.

\section{Method}

In May 2015 the authors discovered a small number of academic custom writing sites that were similar in appearance. While the site names, logos, images and colour schemes of these sites were different, the structure of the sites looked to be remarkably similar. We surmised that it was likely that these sites had something in common and that there might be more sites like them. A reverse Domain Name Server (rDNS) look up was used to see if there were other websites associated with the Internet Protocol (IP) numbers that were associated with the sites we had already found. An rDNS look up is a way of querying the domain name of an IP address, which is the reverse of how websites are usually accessed via a browser. The process can be used to reveal the Universal Resource Locators (URL) of other websites using the same IP numbers. Through this process other sites were identified that clearly related to contract cheating.

Through this process over 130 sites were found that are the type most obviously recognisable as contract-cheating sites. These sites display the persuasive features that Rowland et al. have identified as being typical of academic custom writing sites sites. They divide these features into three categories: informativeness (eg information about services offered and terms of use), credibility (eg assurances of the quality of both the writers and the work produced, as well as of price, of privacy and of payment security) and involvement (eg offering customers 24/7 live text chat services, social media and personal accounts) (Rowland et al. 2017). Many of the sites provide phone numbers with British, American and/or Australian area codes. Toll-free forwarding phone numbers (that allow owners of online businesses to offer clients local phone numbers that are forwarded to a phone line in a different geographic location) make it likely that these sites are not necessarily operating out of one or any of these locations. We call these sites 'client sites' as they are where student 'clients' place orders for academic work to be produced for them.

Through this rDNS process we were surprised to discover two other types of site that were clearly associated with the academic custom writing industry. These are markedly different to these client sites in the features they displayed. The first are sites that invite people to register as writers in order to work to produce custom written essays. We call these 'writer sites' and we found over 30 of these. The persuasive features of these sites typically include assurances of the quantity of work available, the ability for writers to work from home, generous pay (including bonuses) and the availability of a wide range of writing projects in different disciplines. The second are sites that offer to set up, in return for a fee, fully integrated academic writing websites. We call these 'master sites' 
and we found four sites in total that are linked together as two pairs. These sites offer pricing structures, live demonstrations - what they call 'live demos' - and a list of features of the sites they offer to build. One of the key features listed on these sites is that they build two separate types of site: a writer site that also functions as an administrator site and one or more client sites that are linked together and to what they refer to as the 'admin/writer' site. This offered an indiciation that there may have been some kind of link between the client and writer sites already found. Indeed the two pairs of master sites are constructed and joined together in this way in order to demonstrate this structure to potential buyers. The two sites in one pair display identical contact phone numbers and email addresses while the two sites in the other pair display identical email addresses only.

The discovery of these three different types of sites indicate that the structure of academic custom writing sites is potentially more complex and sophisticated than had been previously reported in the scholarly literature. There is a widespread assumption in the literature that what we call client sites constitute a single, self-contained academic custom writing business. These initial findings indicated that this was not the case. Our hypothesis was that the main administrative component of these online businesses is actually the writer sites and that in many instances multiple client sites were all part of the one company.

To find evidence to support this hypothesis initial information was gathered from a variety of sources from the client and writer sites discovered through the rDNS look up within the time frame of a single week in December 2017. This short time frame was used because of the highly volatile nature of these sites; they have a tendency to go into and out of existence frequently. First IP numbers and Internet Service Provider (ISP) names were collected for all the sites. This information was used to identify a total of 37 client sites and 10 writer sites that appeared to cluster around identical IP numbers and ISP names. Then, for each of the 37 client sites, the order process was initiated, stopping just prior to order submission to determine the identifier (eg PayPal identity) of the merchant that would receive payment (had the order been completed). The phone numbers advertised on the sites were recorded. None of the writer sites advertise phone numbers and because they do not need to accept payment, it is also not possible to gather merchant identifiers from them.

In addition, two sets of information from the two pairs of master sites were gathered. The first set was the features list that explains to potential buyers the nature of the products (i.e. separate 'admin/writer' and client sites) that are available for purchase from these sites. The second set was gathered by investigating the so-called 'live demos' that are made available for the purposes of allowing guests to explore the functionality of the client and writer sites. These 'demos' gave access to the features that only the owners and the site administrators of these writer and client sites would normally see. The information gathered from these 'demos' enabled the reverse engineering of the business processes operating within the client and writer sites. This was done by using the guest login user names and passwords advertised on one of the master sites to enter the demonstration sites and then to observe, analyse and record the functions afforded, the business rules enforced and the business roles supported by the website. These were then compared with the features promoted by both master sites. While the features of the master sites differ slightly, the general nature of the functions and business 
processes observed are substantively similar. From this a single business process that operates within the client and writer sites offered for sale from these master sites was documented.

\section{Findings}

\section{Academic custom writing site structure}

Our investigation has confirmed the basic structure of the academic custom writing businesses that we have discovered: at least one writer site accompanied by at least one, and in most instances several, client sites. Multiple client sites that share identical Paypal information, phone numbers, IP addresses and ISP names were found. This makes it highly likely that each of these clusters of client sites is operating as a single business. The fact that these sites all have different domain names but share identical IP addresses indicates that they are making use of shared web hosting, which is a cost effective way of making websites available online and is a further strong indicator of a relationship between these sites.

While it is not possible to definitively prove which writer sites are linked to which clusters of client sites, the fact that the IP and ISP locations shared by these client sites are each shared with at least a single writer site indicates that they too are using the same shared web hosting as the client sites. While it is not possible to definitively prove from this information that the sites are interlinked, the alignment of all of these factors is highly unlikely to be coincidental (see Table 1 and Fig. 1). It is also not possible to definitively prove which, or indeed if any, of the client/writer site combinations have been built by either of the two master sites. However, the fact that the writer and client site structure that they offer to build is also reflected in the interrelationship of client and writer sites found adjacent to them via the rDNS look up corroborates that this is a viable, and probably prevalent, structure for these types of site.

The relationships between the client sites and the writer sites all confirms the likelihood that one or multiple client sites are owned and/or maintained by the same person or company alongside at least one writer site and are operating as separate 'shop windows' for a single business. After all, the more client sites that are linked through to a writer site, the greater the potential for market exposure. This allows a single site owner to cater to distinct markets such as different discipline areas (for instance, sites specifically appealing to nursing or engineering students) or to geographic locations (for instance, sites specifically targeted to British or Australian students). This establishes the illusion of choice and/or specialisation. The client interface allows students to place orders and to communicate with the site staff about their order. The writer sites provide an interface for the freelance writers to be allocated and/or to bid for available orders, and to submit and revise the work they have completed.

\section{Academic custom writing site business processes}

\section{Administration roles}

As the features listed on the master sites explains, the writer sites also function as administrator sites. The analysis of the features list and subsequent investigation of the live demos' confirm that these sites support multiple roles including order evaluation, payment processing, quality assurance, customer service and support, technical support, 


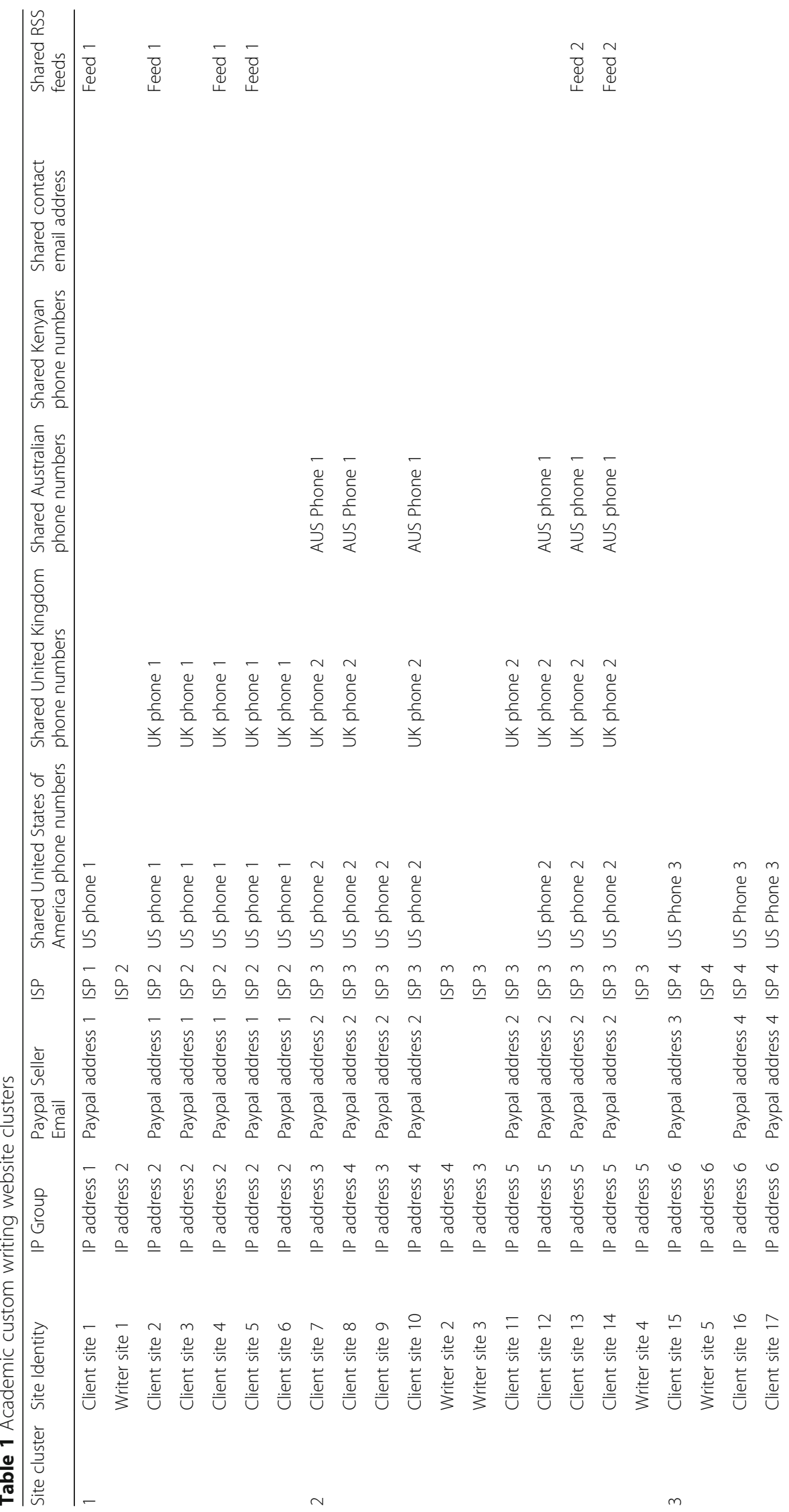




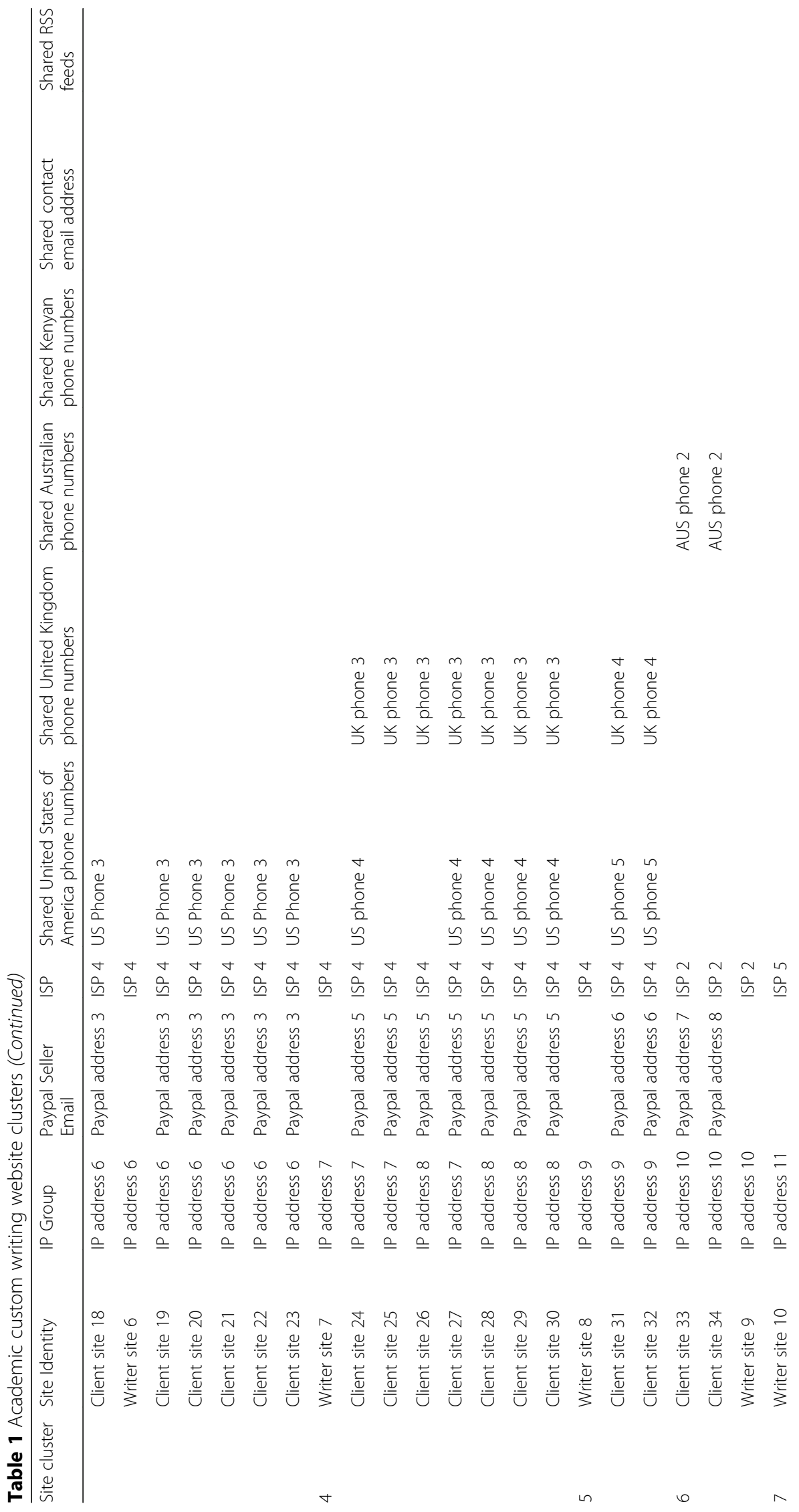




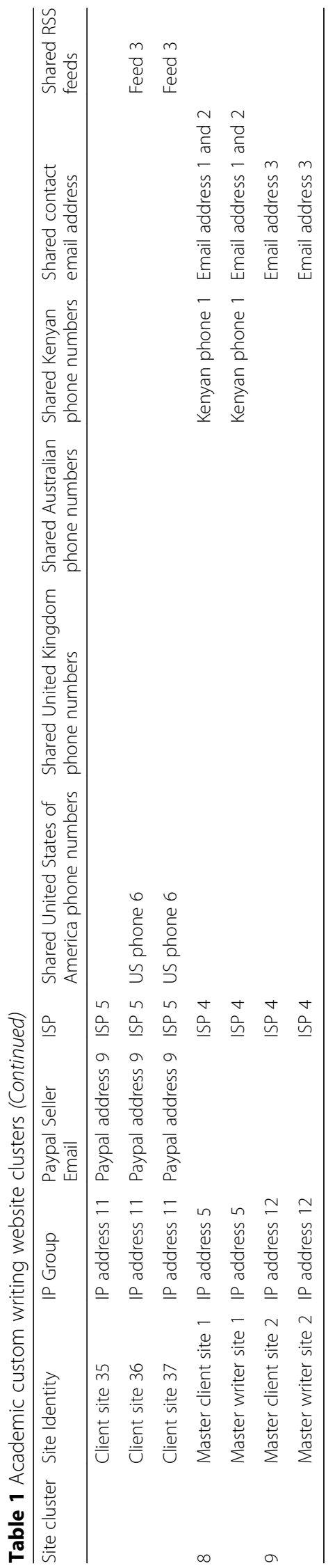




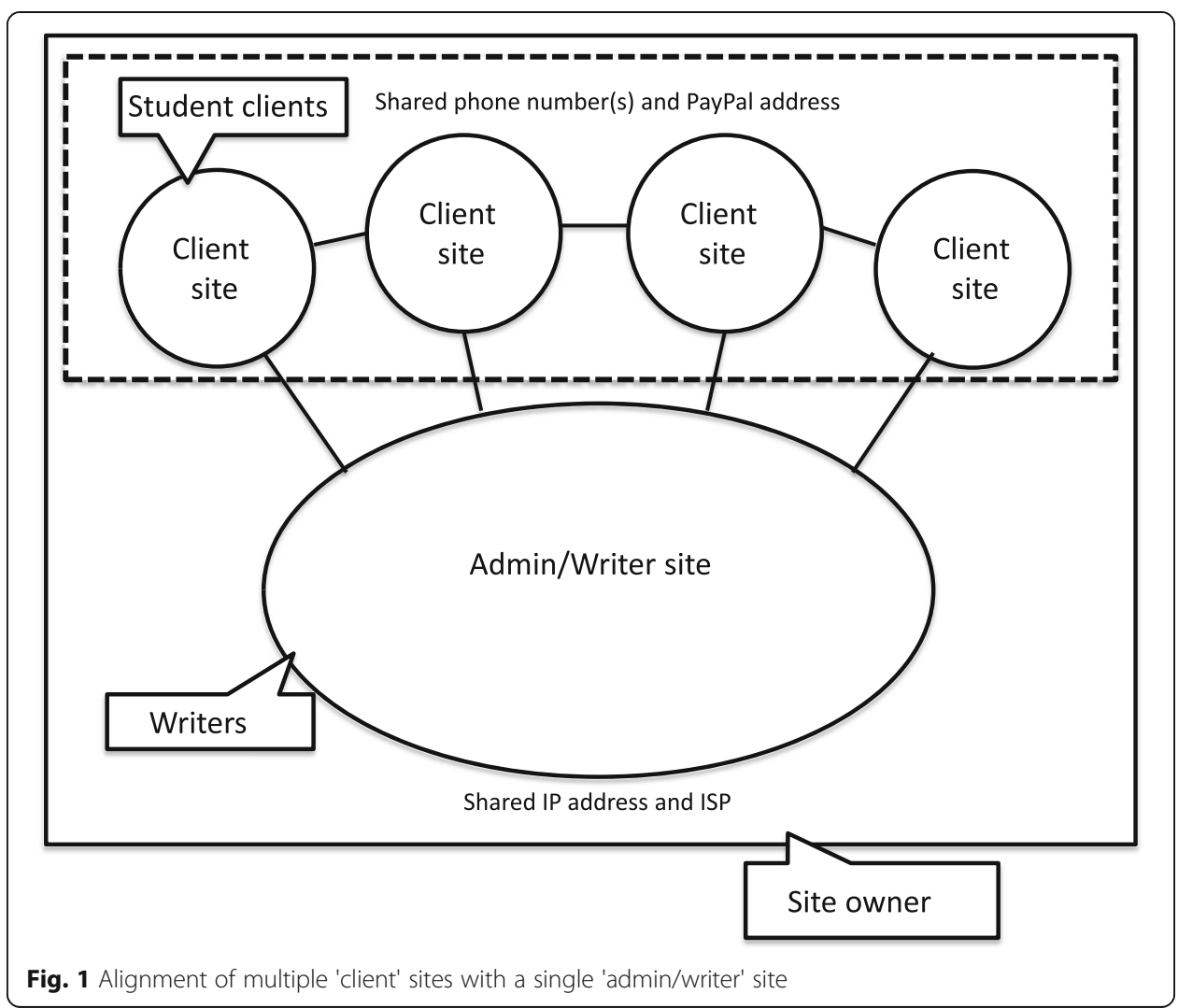

workforce management, and dispute resolution. In a section called 'create new user' the sites allow for each of these roles to be set up so that different people can undertake them separately. There is also an administrator role that is a kind of super-user role, incorporating all of the other other roles into one (see Fig. 2). The fact that the web software enables these roles to be undertaken by one person or, alternatively, by different people in each of the roles indicates that the software has been specifically designed to scale with the owner's business. This is almost certainly to accommodate increasingly higher volumes of orders and growing staff numbers. For the sake of simplicity, in the description of the

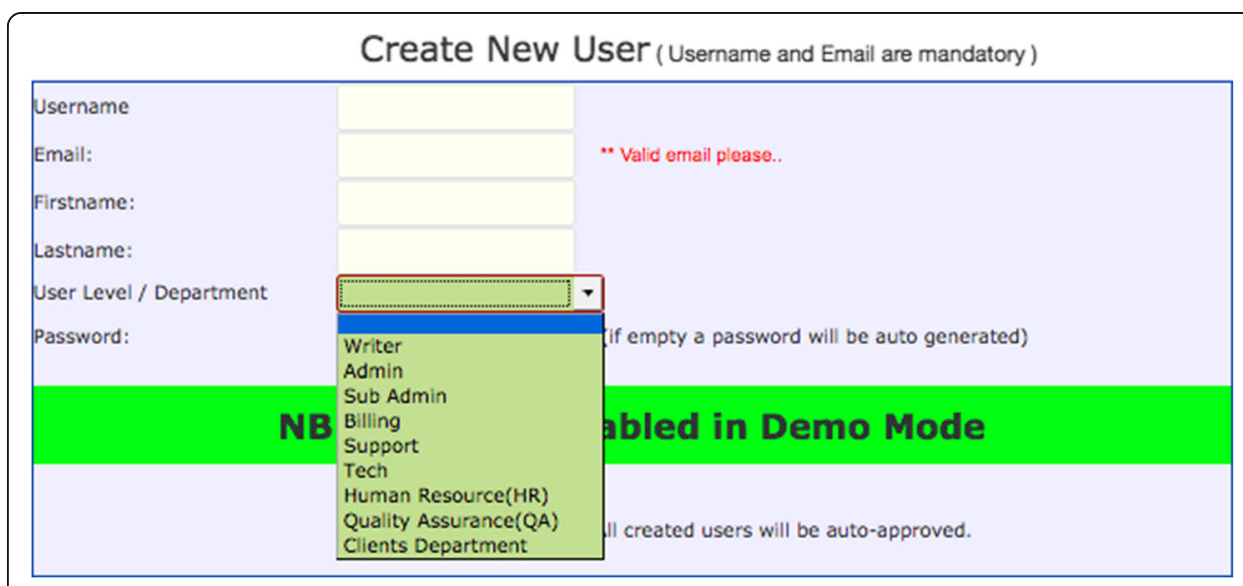

Fig. 2 Screenshot of create new user function in the 'demo' site showing administrative roles 
business roles and processes below, the relevant staff member undertaking a task is referred to as an 'adminstrator'.

\section{Order form}

First this paper turns to explain the process of managing and completing orders as observed and experienced on the client site that is part of the master site that was analysed. On the front page there is a large order button that links through to an order page. Clicking on this starts the process, which subsequently prompts clients to enter a range of information into an order form (see Fig. 3). The order form demonstrated on the two master sites shares many features with those on many but not all of the client sites. First it requires users to key in the topic for the order and then to select all or most of the following from a series of pull down menus and check boxes: the subject (discipline) area, the type of document (eg essay, term paper, dissertation chapter), the number of words which is also measured in pages, the line spacing, the urgency (measured in time), the academic level (ranging from High School to $\mathrm{PhD}$ ), the required number of sources, the referencing style and the preferred language style (US or UK English). Clients are invited to enter information into a window entitled 'order description' which instructs them to 'type your instructions here'. On the live sites, clients are required to enter in their names and contact information and to register with a password for the site. Clients are also given the opportunity to upload additional files at a later stage of their order. The final stage of the ordering process adds up the costing of the variables for the academic work and requests payment from the customer.

There are four variables that affect the price of the order: the discipline, the length of the paper, the urgency and the academic level. The cost variance by discipline on the actual client sites differs from one site to another. On some sites, all disciplines are available at the same rate while on others, some disciplines attract a higher per-page rate that on some sites is referred to as a 'complex assignment charge'. Generally speaking, Science, Technology, Engineering and Mathematics (STEM) subjects like Physics, Chemistry, Aeronautics and Statistics are more expensive than Humanities, Arts and Social Sciences (HASS) subjects like English, History, Business and Sociology. Most sites also offer additional services such as 'VIP support' and 'Written by Top 10 Writers' that incur an extra one-off or per-page fee. Many offer discount vouchers for return customers, for recommending a friend and/or for orders over a set amount that can reduce the final cost. The cost variance across these sites warrants further investigation but is outside the scope of this paper.

\section{Order management}

This paper now turns to explain what happens after the orders have been lodged. This workflow has been represented diagrammatically using a business process diagram that shows the major steps of the order completion from the point of view of the site owner (see Fig. 4). Administrators adjust the status of an order as it moves through the various stages of being filled. On the 'demo' site we analysed, the site administrators are able to adjust the status by selecting an order from a list using a check box and then clicking one of a number of different buttons which include: 'make available,' 'mark as done,' 'return to editing,' 'return to revision,' 'un approve,' 'remove' and 'delete' (see Fig. 5). This is illustrated using a diagram that tracks each of the logical states that an order can take from first inquiry to completion (see Fig. 6). 
Place a new order now! It takes just a few minutes

1. Submit your paper details $\gg 2$. Pay for the order $\gg 3$. Download your custom written paper

Custom Paper Detalls

Topic: *

Subject area: *

Type of document:

Number of

pages/words: *

Spacing:

Urgency:

Academic Level:

Ant

Essay

1 pago/asprox 275 words

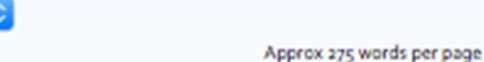

Single spaced

8 nours

High Sehood

Number of sources

Ireferences:

Style:

Please Solect.

Please Solect...

Written by Top 10 Writers

52.95/page

Total VIP support: $\square \mathbf{\$ 5 . 9 5}$

USD 0.00

Preferred language

style:

Track ID

Please Sorect.

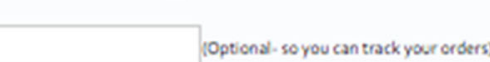

Optond so vou can track your orders)

Order description: *

(type your

instructions here)

If you have additional files, you will upload them at 'Manage Orders' section.

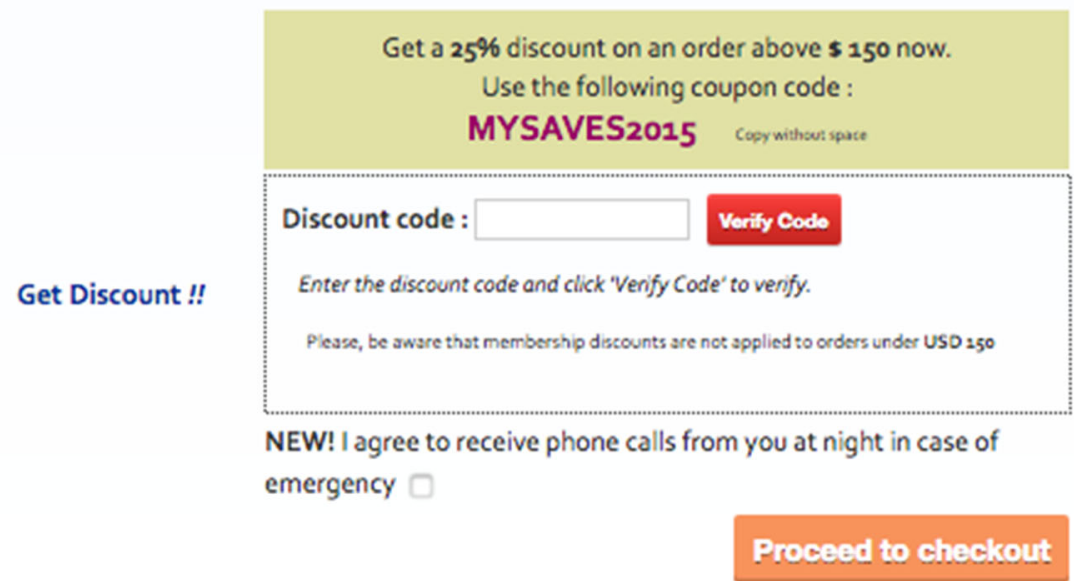

Fig. 3 Screenshot of order form in the 'demo' site 


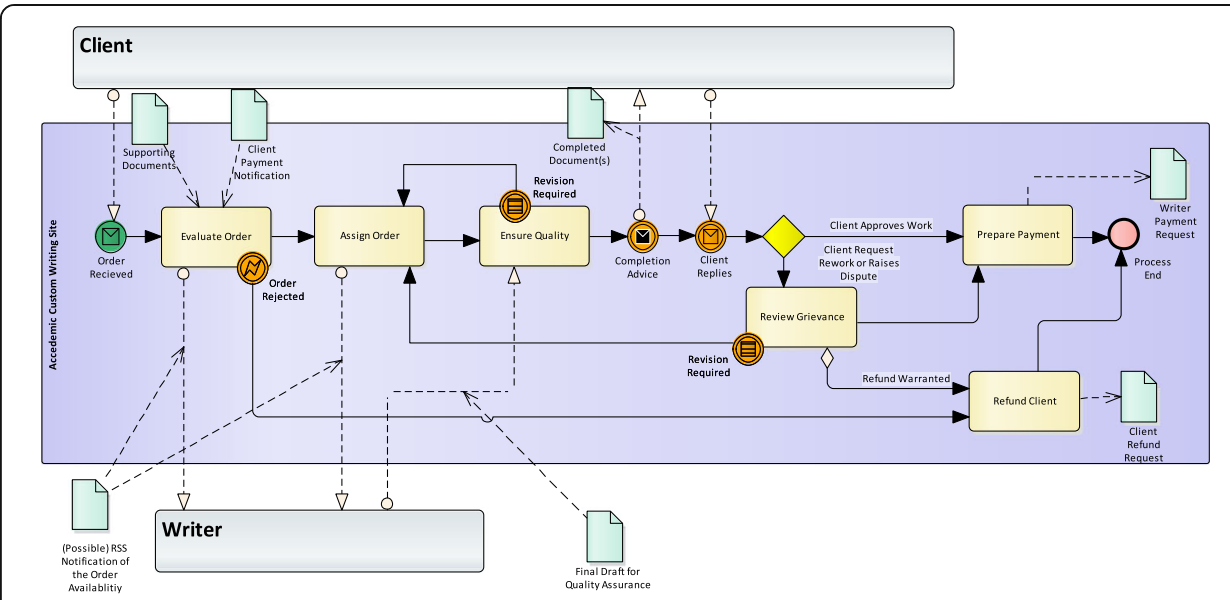

Fig. 4 Business process diagram

When orders are placed on the site they are automatically set to 'pending.' At this point an administrator vets the orders to ensure that they are legitimate and that they contain enough information for a writer to produce the work that is required. If it does not, the order's status is changed to 'rejected'. If it does the order's status is changed to 'available'.

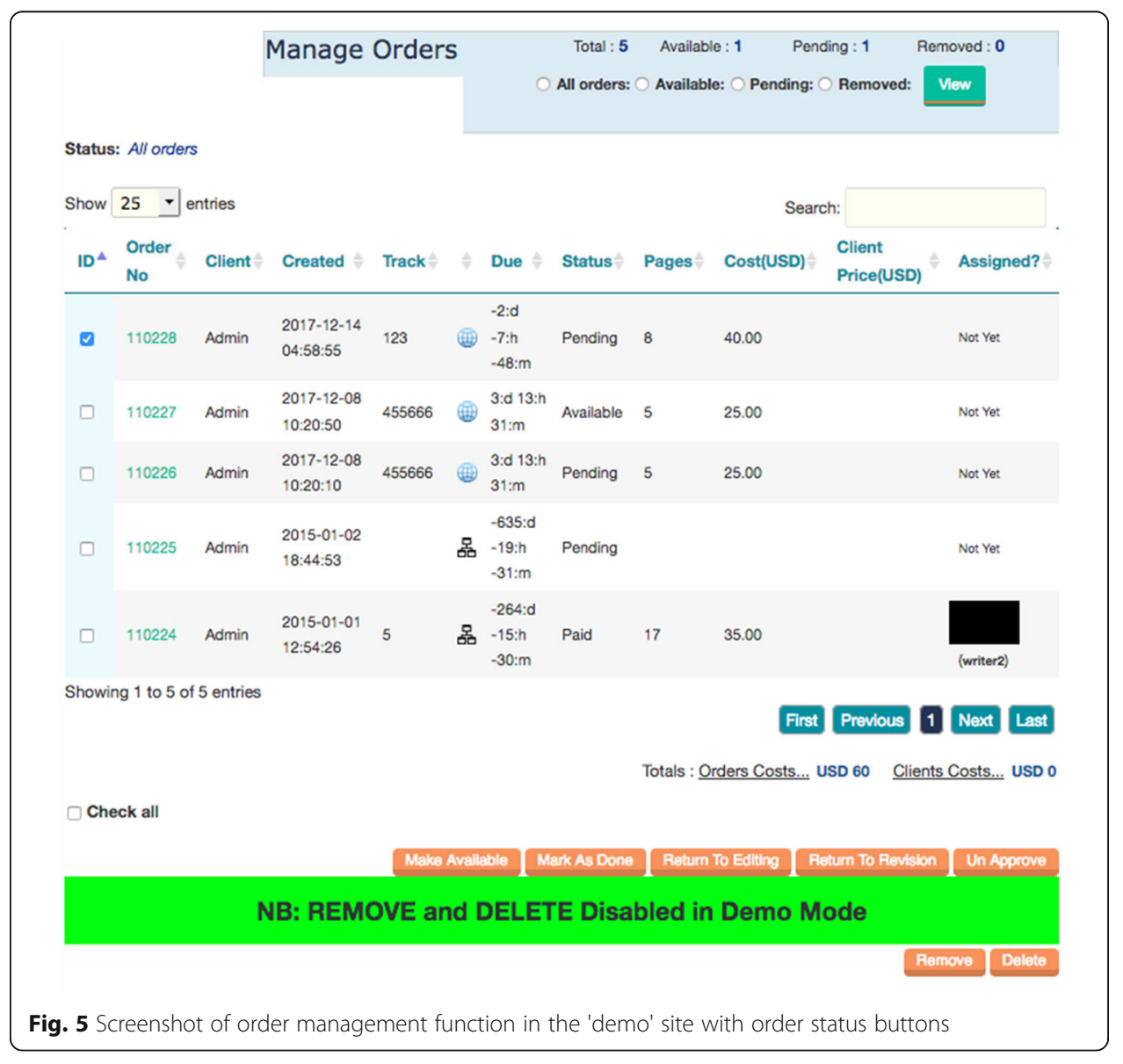




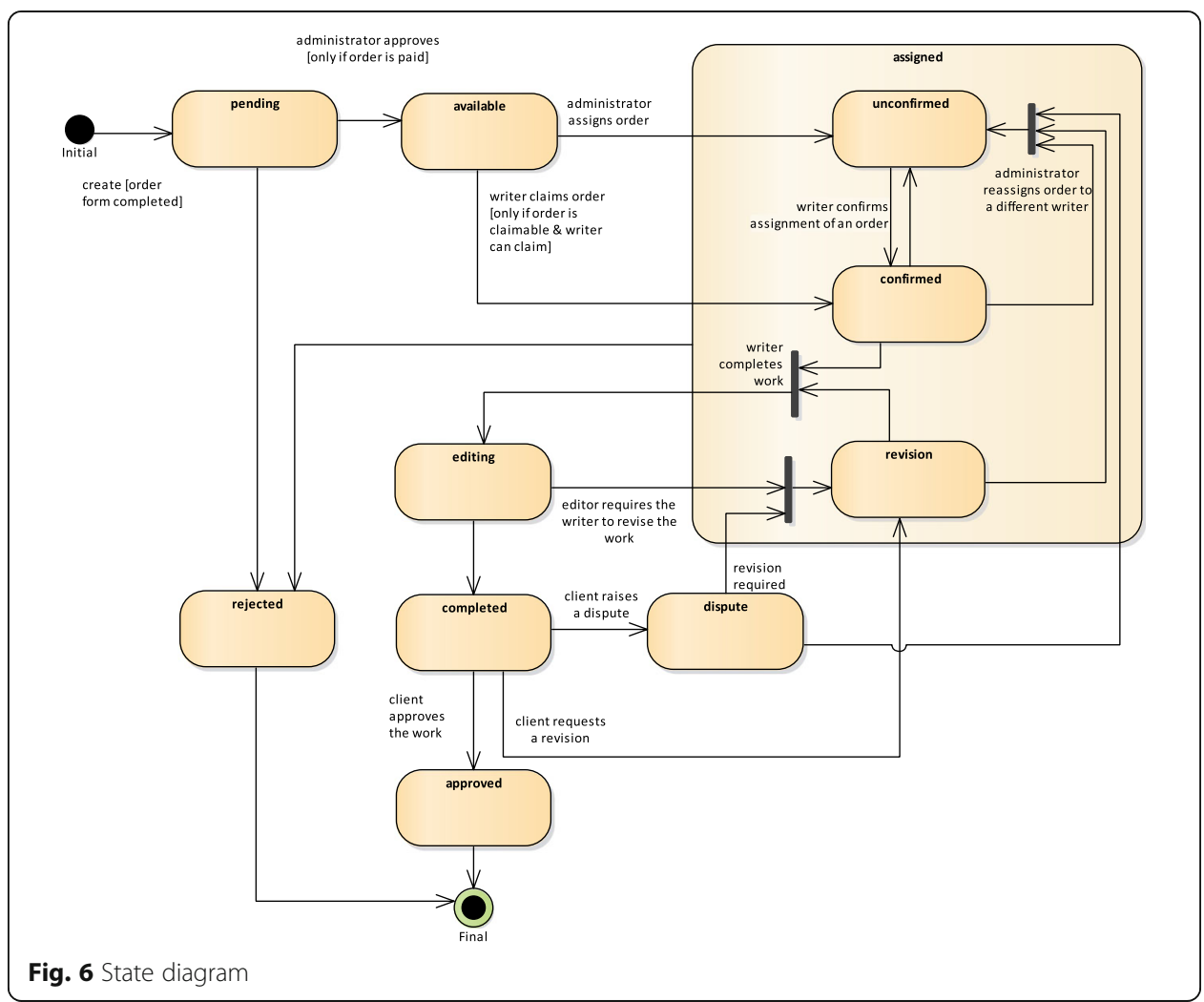

Most client and writer sites have a publicly available Rich Site Summary (RSS) feed that is populated with information relating to orders placed on the site. This function is disabled on the 'demo' (presumably to avoid test orders from being publicly published). The RSS facility is nevertheless available on the master site albeit in an empty state (see Fig. 7). The existence of the RSS feed is not described in the features list of either master site so its precise purpose is not clear. We have inferred how it probably works and how it is populated.

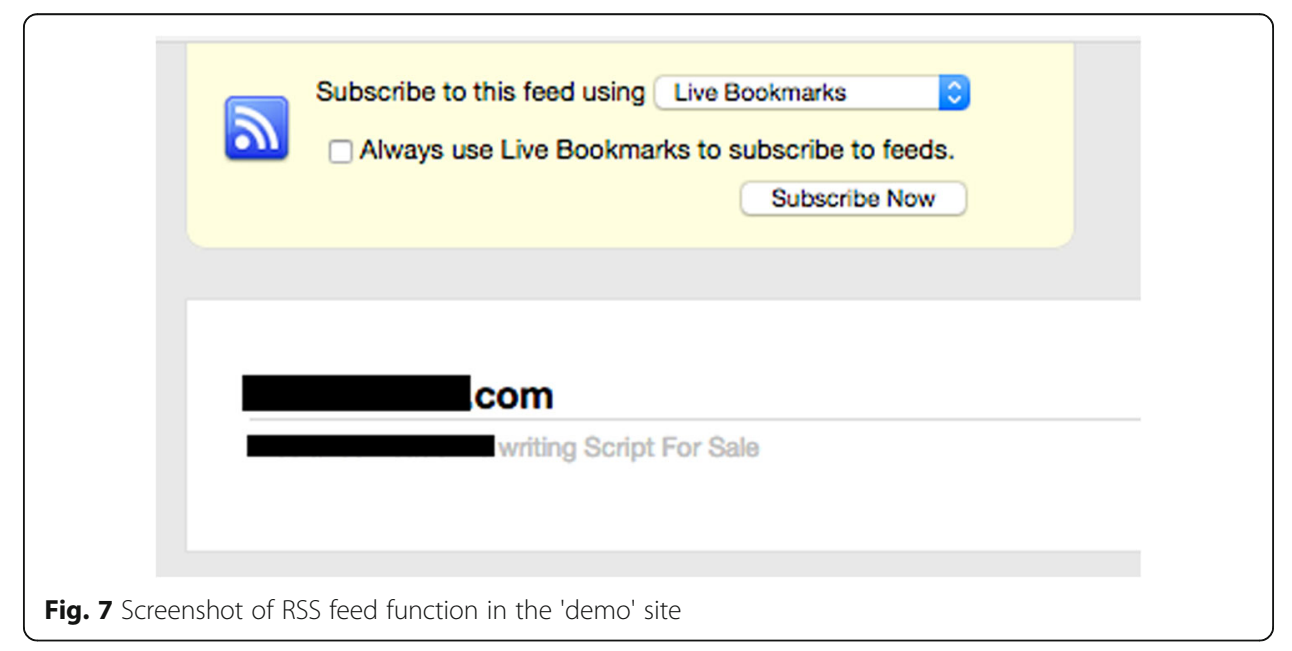


RSS feeds emerged in 1999 as a means of publishing (through aggregation and syndication) frequently updated online content so that readers can easily track it. The fact that the initials RSS are often taken to mean Really Simple Syndication gives an indication of the simple and interoperable format that these feeds take. The logical purpose of the RSS feeds emerging from these sites is to allow writers to maintain easy and immediate access to new orders as they become available, particularly if they are working for multiple companies.

It is possible to be confident that the content emerging from these sites via the RSS feeds relates to orders placed by student clients. There are, however, many aspects of these feeds about which it is not possible to be certain. Prime amongst these is the fact that RSS feeds from a particular site can contain orders that are duplicated in feeds from other sites. There are at least three different possible reasons for these duplications. The first is a result of the interlinked nature of the websites, whereby the one order is distributed (or syndicated) to multiple feeds, presumably in order to make it visible to the largest possible number of available writers (see Table 1). The second is when multiple students in the same course order the same piece of academic work from one or multiple sites. The third is when unclaimed orders (i.e. orders for which a writer has not yet been allocated) and work that has not been started or satisfactorily completed by a previously allocated writer are readvertised via the feed. These orders then become available for reallocation to another writer.

Another thing that cannot be known for certain about these RSS feeds is whether the orders emerging from a site are all the orders that are placed on that site. The date stamps of the feeds cluster closely together which indicates that a staff member working for the site publishes them manually rather than being generated automatically when an order is placed. The most logical moment in the workflow for this to happen is after an order has been vetted and before it has been assigned. It is possible, therefore, that in practice the orders that are pushed to the RSS feeds represent only a subset of the orders that come into the site. This may be because site owners and a select group of writers give themselves the first option to complete orders as they come into the site and it is only the residue of unclaimed orders that is subsequently sent through the RSS feed. From the information contained in these feeds it is not possible to ascertain who has placed the order, exactly when they placed it, at which institution they are studying, whether the order has been filled and, if so, whether it was subsequently submitted to an institution for assessment. What it does make certain is that a student has placed an order for the work to be done.

\section{Bidding and writer status}

As explained on the features list of the master sites, writers are given different levels of access to orders. An administrator can enter an area called 'writers administration page' and select a writer or writers from a list using a check box and then choose the relevant status from a pull down menu. In one master site the writer statuses are set as 'silver', 'gold' and 'platinum' and on the other they are 'normal', 'silver' and 'advanced'. In this same area of the site, the administrator is also able to select writers and click buttons entitled 'approve, 'ban,' 'de activate,' 'delete' and 'reset password' to manage other aspects of a writer's work status and access to the site (see Fig. 8). The features list of both master sites explain that the different status levels are awarded once writers prove the 


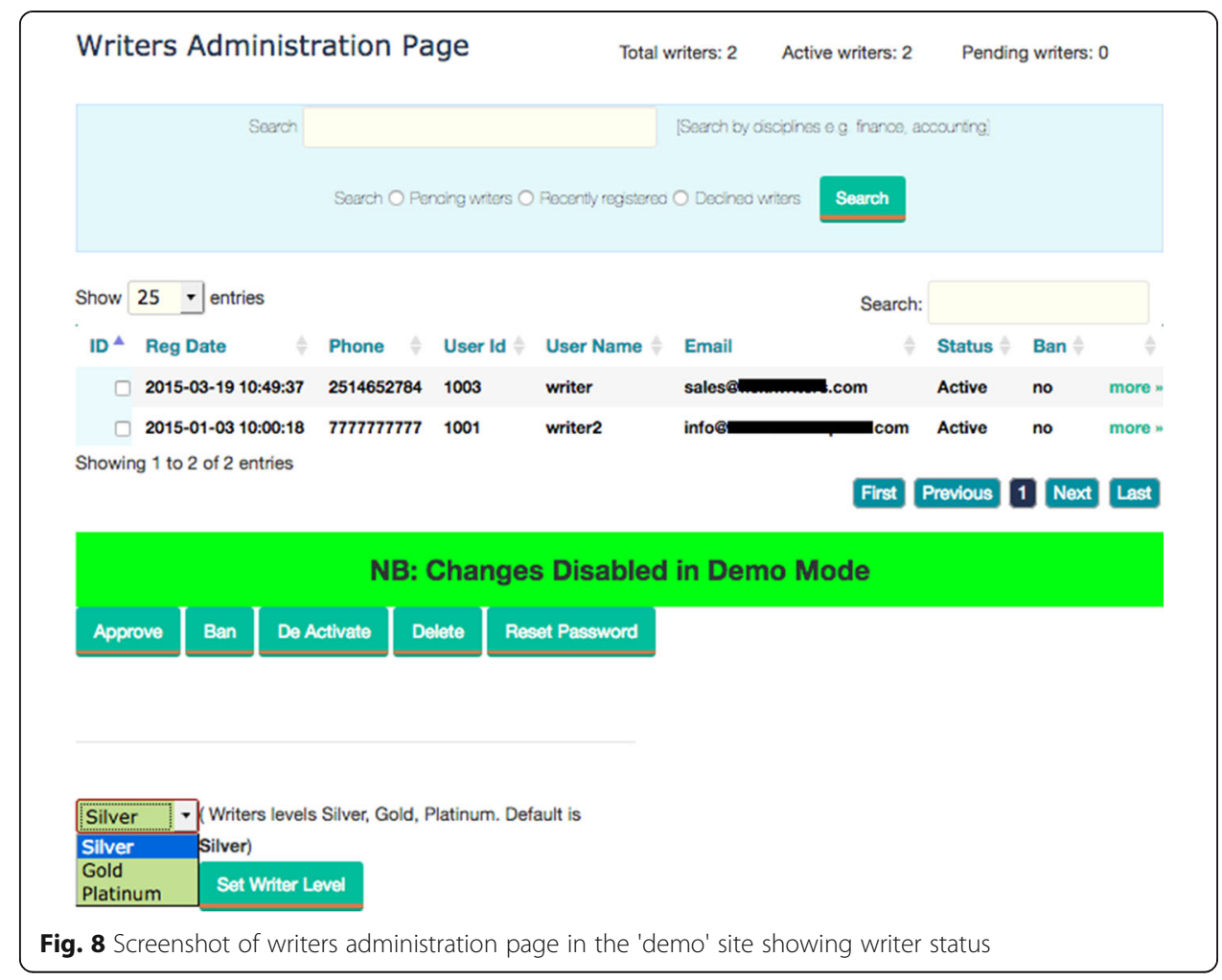

quality of their work. The 'site settings' section allows an administrator to adjust the number of orders for which a writer at each status level is entitled to claim and bid using an edit function (see Fig. 9). Once an order is allocated to a writer its status changes to 'assigned'.

\section{Quality assurance}

Once the writers have completed the work, the writers upload the orders back to the sites. The orders are then put through a quality control process overseen by an administrator. At this point an administrator changes the order's status to 'editing'. If through this quality control process it is deemed that the work requires further revisions it is sent back to the writer for adjustment. At this point its status is changed to 'revision'. If an order is claimed by a writer but not filled on time or to an appropriate level of quality, an administrator can make it available for reallocation to other writers. At this point its status reverts to 'unconfirmed'. Once the completed work has been approved through the quality control process the order's status is changed to 'completed.' At this point it is made available to the client who has ordered the work. If the client is happy with the quality of the work provided, they can confirm the order as completed. At this point its status changes to 'approved'. If they are not happy, they can ask for a revision. At this point the order's status reverts to 'revision'. If they remain unsatisfied after the revision process, they can further escalate their concerns at which point the order's status changes to 'dispute'.

It is useful to note at this point two particularly striking features of these sites. The first is the sophisticated nature of the workflows that operate within them. The second is the meticulous nature of the quality assurance and dispute resolution mechanisms built into the workflow. 


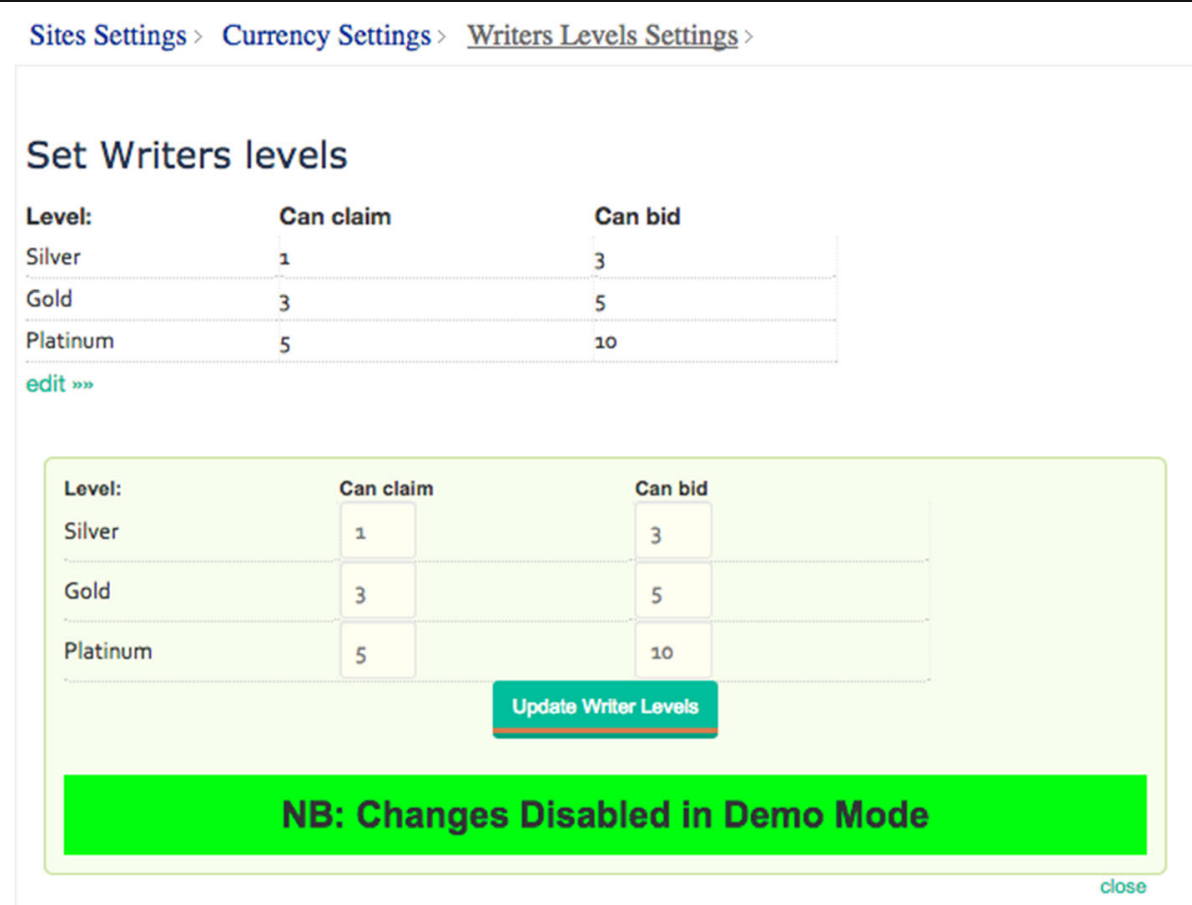

Fig. 9 Screenshot of set writers levels function in the 'demo' site

\section{Discussion}

\section{Business models}

As George and Bock point out, 'definitions for business models vary widely' (George and Bock 2011 p. 83). For the purposes of this paper we use a definition offered by Amit and Zott: 'A business model depicts the design of transaction content, structure and governance so as to create value through the exploitation of business opportunities' (Amit and Zott 2001 p. 495). There are three different business models at work within this particular set of academic custom writings sites.

The first, and simplest, business model is that of the writers are working as selfemployed freelancers. Dave Tomar - who is probably better known by his original pseudonym Ed Dante and by the moniker the Shadow Scholar - has described this business model in some detail in an article (Dante 2010) and subsequent book (Tomar 2012). His choice of the pseudonym 'Dante' is apt as he refers to the working life he experienced as a writer for academic custom writing companies as being his 'personal hell' (Tomar 2012 p. 157). A Chronicle of Higher Education report by Xian $\mathrm{Bu}$ has identified some of these writers as graduates living in Kenya who report earning between USD5000 and USD14 000 per month (which Bu reports compares very favourably to their public service salaries of USD500 per month and against the per capita gross national income of Kenya which is around USD1300) (Bu 2016). Bu's article also reports on a subsidiary aspect to this business model: the onselling of writers' accounts. The fact that the writers are given different status levels, with attendant privileges as outlined above, explains how there is value in an established writing profile once a certain status level has been obtained. 
The second business model - that builds on and amplifies the first - is that of the academic custom writing business owner. This business model creates value by establishing a marketable web presence and then benefits by taking a portion of the revenue generated by client orders. A report in the Chronicle of Higher Education by Brad Wolverton quotes a site owner explaining that he takes $50 \%$ of the revenue and pays the writers the rest (Wolverton 2016). Tomar also reports: 'Almost universally, wherever I have worked, you collect half the money and the company collects the other half' (Tomar 2012 p. 141).

The third and final business model is that of the master site owners who build academic custom writing sites - that is both the admin/writer and client sites - for a fee. This business model - that builds on and amplifies the first and second models - creates value by selling pre-designed, proven and tested web applications with all the business processes built in and the instructions on how to use them for a one-off payment. These master sites design the logos and brands for these websites and also offer webhosting and application management.

\section{Site structure}

Clearly these sites are clustered together with one or multiple client sites linked together with a writer site using the same shared web hosting, PayPal addresses and phone numbers. The fact that these match what is being advertised on the master sites makes it likely that the same person or people built them using the same template. If so, they will also have similar or identical business processes operating within them. This is an example of business process isomorphism, which Masini defines as a 'phenomenon whereby organizations tend to display similar business processes [which] can be associated with the adoption of similar software packages containing similar process templates' (Masini 2009 p. 109). It is possible that the owners of the master sites have designed the software that they sell based on their own experiences as writers within the academic custom writing industry and have modelled their sites on the experiences they have had. It is also likely that people who have previously worked as writers move on to establishing their own businesses (client and writer sites). This enables them to take on a role that may be both less labour intensive and more lucrative. In this role, the site owners probably earn on average $50 \%$ of the revenue for orders completed by other writers. They are also able to reserve easy and lucrative writing orders for themselves for which they earn all of the revenue. These master sites, therefore, cater to individuals who aspire to establish businesses of their own but do not have the skills or time to design and build their own websites.

\section{Speed of production, and quality control and assurance}

A core component of the business model of these sites is their ability to link clients' orders with as many writers possible. From the site owner's point of view, this is desirable because it makes it more likely that the order will be filled in the required time frame, thereby satisfying the client. The exposure of each order to as many writers as possible also increases the competition for the orders amongst the writers. This reduces the cost of production, thereby making the commodity more affordable and attractive to potential clients. Because of the fixed pricing model, the site administrators are able to secure the bulk of the revenue regardless of where the bidding lands amongst writers. 
The extensive quality control and assurance processes built into the workflows of these businesses indicates how important it is for these sites to produce work to a level of quality that satisfies their clients. The specialised roles of quality assurance, customer service and support, and dispute resolution that are provided for in these sites are further indications of the importance of customer satisfaction for this business model. As Lines confirms, the quality of the work being produced by the sites she investigates is literally of a passable standard (Lines 2016 p. 18). Ensuring the quality of work being produced by these sites is crucial to maintaining two of the most important sources of new work: return customers and their personal recommendation to new clients.

From the writers' point of view, it is in their best interests to seek out orders that they find easy to fill (which will vary depending on their disciplinary expertise and previous writing experience) and which will be lucrative. In his book Tomar describes his approach to stockpiling orders:

I would grab up the paper, and it would go on top of the huge, miserable pile of shit that I had to shovel through. [...] As soon as I finished one paper, I started another. As soon as I submitted an assignment, I went to the writers' board and picked up another one to take its place. (Tomar 2012 p. 174-5).

$\mathrm{Bu}$ also offers a description of the stockpiling approach taken by 'Solomon', one of the Kenyan writers he interviewed for his article. It is strikingly similar to Tomar's description: 'Orders come through the companies' websites, and sometimes a dozen writers will vie for one. Whoever acts fastest gets it' (Bu $2016 \mathrm{np}$ ). This practice is illustrated by an image in Wolverton's article of a handwritten list of orders 'stockpiled' by a writer, with the caption: 'a professional cheater's little black book of assignments' (Wolverton $2016 \mathrm{np}$ ). It is clear from these descriptions that the practice of stockpiling orders by writers to secure a reliable stream of income is fairly standard. As explained earlier, in the sites examined in this paper, the writers have to earn their right to claim and stockpile work in this way. For the writers to have the best chance of spotting and claiming the orders that best suit their skills they need to maintain easy, constant access to orders as they become available. This is the role of the RSS feed.

The feed saves writers the time and trouble of regularly logging in to one or multiple writers' sites to check for new orders. Instead, the writers probably subscribe to the feeds and use an RSS reader to keep track of new orders as they become available in real time. Being able to access these feeds makes it much easier for writers to work for multiple sites. The feed, therefore, provides site owners with a quick, easy and cheap mechanism for advertising available orders to a wide pool of writers. It also provides writers with a quick, easy and cheap mechanism for monitoring orders and claiming them as quickly as possible. Wallace and Newton found that "requested turnaround times for contract cheating are, on average 5 days, and there appears to be a large capacity for shorter turnaround times to be achieved" (Wallace and Newton 2014 p. 235). The fact that orders with short 'urgency' times are the most lucrative and the existence of these RSS feeds that allow writers to bid quickly for orders together offer a compelling explanation as to why the turnaround times can be so short.

In the first decade of the twenty-first Century when Tomar was working as a writer in the academic custom writing industry the barriers to entering the market as a site 
owner were higher than they are now. The technical expertise required to establish a website and manage the eCommerce of such an enterprise meant that the sector probably had a much smaller number of sites. Now, as explained above, the master sites will set up the complete package thus eliminating the need to have any coding skills. These sites also facilitate web hosting and management services alongside a tried and tested set of business processes. The skills required to operate and administer these sites once they are established require only basic computer literacy and business acumen. Companies like PayPal have also made it much easier for clients and site owners to manage foreign currency transactions.

\section{Conclusions and recommendations}

As indicated at the outset of this paper, in order to improve the deterrence and detection of contract cheating, it is important to understand better the problem. To that end, the information and findings offered in this paper provide new information to academics and institutions about the business processes and models that sit behind some academic custom writing sites. These sites make up one part of one segment of the contract-cheating problem as a whole.

The business models that are supported by the sites investigated here appear to be well established and mature. This indicates that there is a substantial market that is feeding this industry. The sophisticated quality assurance processes built into the workflows supported by these sites indicate how important the quality of the work produced within them is to all three business models. This shows how important return business and word-of-mouth recommendations are but also how likely it is that the quality of the work being produced by the sites is, at least, of a passable standard. Alongside this, the existence of the RSS feeds indicates the importance of speed and efficiency to the industry. Together, these confirm that the priority for both the site owners and their student clients is for the orders to be filled on time and to good enough quality. For future research on these sites, it is important to be careful not to assume that different sites are running as separate businesses; in some instances this may be the case but in other instances they may simply be different 'shop windows' for the same company.

Finally, this paper offers some advice about practical things every academic and institution can do to improve their chances of both detecting and deterring contract cheating. In terms of detection, the fact that the RSS feeds that come out of the client sites are publicly available means that they can be picked up by a web search. The RSS feeds frequently contain text provided by students, which is often the exact wording of assessment briefs that have been copied by them directly onto the order forms. Academics who routinely undertake a web search using text taken from their assessment briefs are likely to be able quickly and easily to identify if orders for work have been placed on these academic custom writing sites. While this will rarely provide enough information to identify any one particular student who has ordered the work, it will alert academics to be vigilant for it amongst the work that they are marking. This suggests that to be effective as a deterrence tool, academic staff responsible for marking student work could benefit from information, support and training on what to look out for and how act to on anything that raises their suspicions (see Dawson and Sutherland-Smith 2017). 
In the, albeit limited, literature on contract cheating, one proposed solution to the problem is to limit the time span between releasing the assessment brief and the due date (Mahmood 2009; O'Malley and Roberts 2011). Even though students who are procuring work with days or even hours to spare are spending more money than their better organised peers who place orders weeks in advance, there is evidence that writers seek these orders out and compete with other writers for them because they provide the more lucrative work. The sad reality is that a lot of the work that is being produced by academic custom writing sites is being churned out very quickly indeed. As noted by Wallace and Newton (2014) reducing the turnaround time is unlikely to prevent or deter contract cheating using these sites and may even encourage it by placing extra pressures on students.

The covert nature of this industry means that their advertising outlets are relatively limited. It is clear from our analysis that the sites investigated here rely heavily on return business but also from word-of-mouth recommendations from their clients. As a result, it is likely that students (including those who have not used and never intend to use the services of these sites) are very aware of contract cheating being undertaken via academic custom writing sites. Institutions need to consider making it easier for students to report any concerns they have about the behaviours of their peers. It might also be wise to provide mechanisms and support for students undertaking group work to report any suspicions they have of someone in their group using an academic custom writing site, and to remind them of the potential damage it could do to all of them. It is also important that academic staff take responsibility for having conversations with their students about the problem of contract cheating. The topic of one such conversation may be to let students know that the privacy and security of these sites is not as robust as they may imagine, given that the RSS feeds routinely post publicly available information about orders made to the site.

In order to have these conversations, it is first vital that all academic staff and the institutions for which they work become aware of and better understand the problem. We all need to be more vigilant in our efforts and that means acknowledging that it is happening and making serious efforts to socialise this knowledge and talk about it with our teaching colleagues.

\section{Acknowledgements}

The authors would like to acknowledge Yan Liu who identified some investigative strategies early in our analysis, Nick Wailes and Alex Steele who offered advice in the early drafting of this paper, David Tittle who provided advice on data management and Scott McBride who assisted with the collection of PayPal and phone number information.

Funding

There are no sources of funding for this research.

Authors' contributions

CE is an Associate Professor in the Faculty of Arts and Social Sciences at UNSW, Sydney. She analysed the content and structure of the websites and wrote the manuscript with IZ and DR. IZ is an Educational Developer who undertook a research assistant role in the Faculty of Arts and Social Sciences at UNSW, Sydney. He undertook the rDNS look up to find the sites, has collected and analysed the RSS data. He also wrote the manuscript with CE. DR is a freelance Business Analyst who is currently working for Transport NSW. He collected the ISP and IP information, analysed the business processes in the master sites and constructed the business process diagrams. All authors read and approved the final manuscript. 


\section{Publisher's Note}

Springer Nature remains neutral with regard to jurisdictional claims in published maps and institutional affiliations.

\section{Author details}

${ }^{1}$ Faculty of Arts and Social Sciences, UNSW, Sydney, NSW 2052, Australia. ${ }^{2}$ Freelance Business Analyst, Sydney, NSW, Australia.

Received: 8 September 2017 Accepted: 26 December 2017

Published online: 11 January 2018

\section{References}

Amit R, Zott C (2001) Value creation in E-business. Strateg Manag J 22:493-520. https://doi.org/10.1002/smj.187 Bartlett T (2009) Cheating Goes Global as Essay Mills Multiply. Chron High Educ Wash 55(A1):A22+

Bertram Gallant T (2008) Academic integrity in the twenty-first century: a teaching and learning imperative. ASHE High Educ Rep 33:1-143. https://doi.org/10.1002/aehe.3305

Bu X (2016) Contract Cheating's African labor. Chron High Educ Wash

Clarke R, Lancaster T (2006) Eliminating the successor to plagiarism? Identifying the usage of contract cheating sites. In: proceedings of 2nd international plagiarism conference

Clarke R, Lancaster T (2007) Establishing a systematic six-stage process for detecting contract cheating. In: 2nd international conference on pervasive computing and applications, pp 342-347

Clarke R, Lancaster T (2013) Commercial aspects of contract cheating. In: proceedings of the 18th ACM conference on innovation and technology in Comput Sci Educ, pp 219-224

Dante $\mathrm{E}$ (2010) The shadow scholar: the man who writes your students' papers tells his story. Chron High Educ Wash

Dawson P, Sutherland-Smith W (2017) Can markers detect contract cheating? Results from a pilot study. Assess Eva High Educ 43:286-293. https://doi.org/10.1080/02602938.2017.1336746

Draper MJ, Ibezim V, Newton PM (2017) Are essay Mills committing fraud? An analysis of their behaviours vs the 2006 fraud act (UK). Int J Educ Integr 13:3. https://doi.org/10.1007/s40979-017-0014-5

George G, Bock AJ (2011) The business model in practice and its implications for entrepreneurship research. Entrep Theory Pract 35:83-111. https://doi.org/10.1111/j.1540-6520.2010.00424.x

Jacks T (2016) Deakin University students kicked out for "contract cheating". The Age

Lancaster T, Clarke R (2014a) An initial analysis of the contextual information available within auction posts on contract cheating agency websites. In: Advanced information networking and applications workshops (WAINA), 2014 28th international conference on. IEEE, Los Alamitos, pp 548-553

Lancaster T, Clarke R (2014b) An observational analysis of the range and extent of contract cheating from online courses found on agency websites. In: complex, intelligent and software intensive systems (CISIS), 2014 eighth international conference on. IEEE, pp 56-63

Lines $L$ (2016) Ghostwriters guaranteeing grades? The quality of online ghostwriting services available to tertiary students in Australia. Teach High Educ 0:1-26. https://doi.org/10.1080/13562517.2016.1198759

Macdonald R, Carroll J (2006) Plagiarism - a complex issue requiring a holistic institutional approach. Assess Eval High Educ 31:233-245. https://doi.org/10.1080/02602930500262536

Mahmood Z (2009) Contract cheating: a new phenomenon in cyber-plagiarism. Commun IBIMA 10:93-97

Masini A (2009) ERP-driven performance changes and process isomorphism. In: Handbook of research on Enterprise systems, pp 96-109

Newton PM, Lang C (2015) Custom Essay Writers, Freelancers and Other Paid Third Parties, pp 1-16. https://doi.org/10.1007/978-981-287-079-7_38-1

O'Malley M, Roberts TS (2011) Plagiarism in science education: preventing cheating via online auctions. In: Proceedings of the Australian conference on science and mathematics education (formerly UniServe science conference)

Rigby D, Burton M, Balcombe K et al (2015) Contract cheating \& the market in essays. J Econ Behav Organ 111:23-37. https://doi.org/10.1016/j.jebo.2014.12.019

Rowland S, Slade C, Wong K-S, Whiting B (2017) "Just turn to us": the persuasive features of contract cheating websites Assess Eval High Educ:1-14. https://doi.org/10.1080/02602938.2017.1391948

Sivasubramaniam S, Kostelidou K, Ramachandran S (2016) A close encounter with ghost-writers: an initial exploration study on background, strategies and attitudes of independent essay providers. Int J Educ Integr 12:1. https://doi.org/10.1007/s40979-016-0007-9

Tomar D (2012) The shadow scholar: how I made a living helping college kids cheat. Bloomsbury, New York

Wallace MJ, Newton PM (2014) Turnaround time and market capacity in contract cheating. Educ Stud 40:233-236. https://doi.org/10.1080/03055698.2014.889597

Wolverton B (2016) The new cheating economy. Chron High Educ Wash 